University of Texas at El Paso

ScholarWorks@UTEP

4-2007

\title{
Fern: An Updatable Authenticated Dictionary Suitable for Distributed Caching
}

\author{
Eric Freudenthal \\ The University of Texas at El Paso, efreudenthal@utep.edu \\ David Herrera \\ The University of Texas at El Paso, daherrera@utep.edu \\ Steve Gutstein \\ Ryan Spring \\ Luc Longpre \\ The University of Texas at El Paso, longpre@utep.edu
}

Follow this and additional works at: https://scholarworks.utep.edu/cs_techrep

Part of the Computer Engineering Commons

Comments:

UTEP-CS-06-45a

\section{Recommended Citation}

Freudenthal, Eric; Herrera, David; Gutstein, Steve; Spring, Ryan; and Longpre, Luc, "Fern: An Updatable Authenticated Dictionary Suitable for Distributed Caching" (2007). Departmental Technical Reports (CS). 215.

https://scholarworks.utep.edu/cs_techrep/215

This Article is brought to you for free and open access by the Computer Science at ScholarWorks@UTEP. It has been accepted for inclusion in Departmental Technical Reports (CS) by an authorized administrator of ScholarWorks@UTEP.For more information, please contact Iweber@utep.edu. 


\title{
Fern : An updatable authenticated dictionary suitable for distributed caching
}

\author{
Eric Freudenthal, David Herrera, Steve Gutstein, Ryan Spring, and Luc Longpré \\ \{efreudenthal, daherrera, smgutstein, rcspring, longpre\}@utep.edu \\ University of Texas at El Paso \\ Computer Science Technical Report \#UTEP-CS-06-45-A \\ December 2006 \\ Updated April 2007
}

\begin{abstract}
Fern is an updatable cryptographically authenticated dictionary developed to propagate identification and authorization information within distributed systems. While conventional authenticated dictionaries permit authorization information to be disseminated by untrusted proxies, these proxies must maintain full duplicates of the dictionary structure. In contrast, Fern incrementally distributes components of its dictionary as required to satisfy client requests and thus is suitable for deployments where clients are likely to require only a small fraction of a dictionary's contents and connectivity may be limited

When dictionary components must be obtained remotely, the latency of lookup and validation operations is dominated by communication time. This latency can be reduced through the exploitation of localitysensitive caching of dictionary components. Fern dictionary's components are suitable for caching and distribution via autonomic scalable locality-aware Content Distribution Networks (CDNs) and therefore can provide these properties without requiring the provisioning of a dedicated distribution infrastructure.

Since search operations on trees are necessarily serialized, communication latency in distributed systems can be tens to thousands of milliseconds, and bandwidth can be limited, the constants matter. Others have recently proposed the construction of incrementally distributed authenticated dictionaries embedded into search structures of logarithmic depth. However, these alternatives require either the sequential transfer of two-to-three times more vertices or the replacement of a greater number of already distributed vertices when updates occur.

A prototype implementation of Fern has been constructed that utilizes the CoralCDN scalable, localityaware, and autonomic content distribution network. We provide an analysis of bandwidth requirements for the Fern authenticated dictionary that corresponds with experimental results. We are not aware of other implemented systems with similar properties or comparable analysis of such systems' performance and bandwidth requirements.
\end{abstract}

\section{Introduction}

Reliable and consistent dissemination and revocation of access permissions and identity mappings are persistent problems in distributed systems. Consistent dissemination of trust and authorization information include online validation services such as OCSP[15] and the dissemination of time-limited public-key based certificates $[15,4,18,11]$. These approaches share a high computational cost for public-key cryptography. They also reflect a range of scalability, timeliness, and connectivity characteristics. On one extreme, on-line status verification protocols provide per-transaction validation of access permissions when sufficient computational and communication bandwidth to trusted online servers can be assured. Credentials with extended lifetimes reduce the bandwidth and computational power required to convey information needed for autho- 
rization decisions by effectively relaxing consistency. However, a window of TOCTTOU ${ }^{1}$ vulnerability is created if no mechanism is incorporated to disseminate and respond to premature revocation.

Updatable online authenticated dictionaries[19, 10, 9, 2] have recently been proposed as complementary source of authorization information based upon integrity-guaranteed name-to-value mappings stored within indexed or searchable data structures. These mappings may be used to provide evidence of authorization or revocation ${ }^{2}$. Composed cryptographic hashes are embedded within these dictionaries in a manner that permits their distribution via untrusted proxies to security-sensitive clients who can efficiently validate mappings (or the lack of mappings) stored within them.

A feature of authenticated dictionaries containing such composed hashes is that the integrity of the entire structure can be witnessed by a single public-key root certificate. This property can be exploited by proxies and security-sensitive clients that incrementally obtain and validate portions of their search structure as required to answer queries. Furthermore, these partial copies of the dictionary's structure can be cached and therefore utilized to answer future queries that share common components.

Modifications to mappings stored within an authenticated dictionary typically cause changes to the dictionary's search structures. Since components of an authenticated dictionary may be cached, it is desirable if these updates are localized. Thus, algorithms that rely on structural modification to achieve good performance (e.g. self-balancing trees) can be problematic.

Fern utilizes a randomized prefix-trie that has expected $O(\log n)$ complexity for all update, lookup, and validation operations. This complexity is asymptotically equivalent to other authenticated dictionaries. However, we argue that, given the high latency of inter-host communication in distributed systems, the constants do matter and Fern's expected cost is comparable to the most aggressive algorithms based upon self-balancing trees and far lower than skip-lists. Furthermore, Fern's algorithm is far simpler than selfbalancing trees and does not require rotation.

While others have proposed designs for authenticated dictionaries whose contents are distributed via an autonomic content distribution network $(\mathrm{CDN})$, we are unaware of any other implemented systems. We present measurements collected from our prototype implementation that utilize the CoralCDN self-organizing and locality-aware content distribution network.

Finally, we believe that we are the first to consider the impact of cacheability upon the cost of monitoring a set of mappings stored within an authenticated dictionary that exploits caching. In Section 4.1, we provide an informal analysis that agrees with observed behavior.

\section{Related Work}

On-line authenticated dictionaries disseminate the contents of a database containing name:value mappings to network-connected clients. The originator of an authenticated dictionary embeds a set of immutable name:value mappings within a search structure ${ }^{3}$. Like other authenticated dictionaries, Fern exploits the structural composition of cryptographic hashes (see Merkle[14]) to amortize the cost of a single public-key crytpographic operation that serves as a witness to the entire dictionary's integrity. This facilitates the scalable distribution of these mappings by untrusted publishers whose clients request and validate copies of mappings stored in these dictionaries by querying any publisher claiming to have a current copy.

Due to their ability to scale to thousands of hosts and respond to dynamic changes in connectivity, we are particularly interested in authenticated dictionary structures that are suitable for dissemination by peer-to-peer content distribution networks. Our prototype of Fern utilizes the CoralCDN[5] locality-aware content-distribution network.

It is desirable for authenticated dictionaries to be structured in a manner that permits (1) publishers to determine the integrity of nodes they distribute and (2) clients to determine the validity of name:value mappings without requiring transfer and/or storage of the entire dictionary structure.

\footnotetext{
${ }^{1}$ Time-of-check-to-time-of-use (see [7])

${ }^{2}$ E.g. that the person known to the dictionary's originator as "Alice" is associated with public key $K_{A}$, or that certificate number $N$ has been revoked.

${ }^{3}$ Fern can also implement a common variant of authenticated dictionary used to communicate certificate revocation lists that disseminates sets of names (e.g. certificate serial numbers) with empty values. Their function is analogous to the word lists frequently used to arbitrate disputes in the popular parlor game Scrabble.
} 
Fern's key-to-value mappings are stored within a search-DAG (directed acyclic graph). Following the model of Merkle's practical digital signature system[14] each vertex of this DAG (including its root) contains a cryptographic hash of its immediate children, and can transitively witness the integrity of all descendents. Thus, path consisting of vertices from such a DAG's root to a vertex containing the referenced mapping serves as a Validation Object (VO)[12] that witnesses the mapping's integrity. The number of nodes in a VO is bounded by the DAG's depth, which is typically logarithmic in the number of mappings stored within the dictionary.

While cryptographic hashes can be used to demonstrate that (portions of) a previously composed data structure has not been corrupted, other mechanisms are typically used to disseminate identifiers for and hashes of updated structures. The identifier permits the dictionary's originator to specify the root of a dictionary structure, and the cryptographic hash provides a witness to the dictionary's integrity. Fern conjoins search-structure identifers and their hashes into a single node identifier (NID) and the root's NID is embedded within a root certificate that both references and authenticates the current root of a Fern dictionary. Like other authenticated dictionaries, the integrity of a Fern root certificate is witnessed by a digital signature generated using asymmetric cryptography.

The typical application of an online authenticated dictionary has a single (exclusive) writer and multiple (concurrent) clients and thus has semantics analogous to the CREW (concurrent-read exclusive-write) shared memory storage model. However, due to an authenticated dictionary's pervasive use of hash composition, it is not sufficient to modify a name:value mapping in place. As a result, authenticated dictionaries are typically immutable and thus provide semantics similar to WORM (write-once, read-many) storage devices. Analogously to hardware WORM devices (e.g. PROM, CD-R), the distribution of updates to dats stored within these structures can be complex or expensive. Originators of such structures typically distribute (1) full replacement dictionaries and/or (2) a continuous stream of updates that must be applied to the previously distributed full dictionary. The first approach can require the periodic dissemination of a large data structure that can be problematic for bandwidth-limited deployments. The second approach requires that publishers obtain all updates since the current dictionary was published and the transmission of all missed updates after a prolonged disconnection can be be expensive. Furthermore, this full-replication approach will distribute portions of the dictionary that may not be referenced by a particular publisher's clients.

In contrast, Fern achieves CREW semantics by permitting originators to distribute in-place updates by replacing and distributing . on demand only those vertices whose contents have changed. Since vertex contents include cryptographic hashes whose value is dependent on their descendants, each updated mapping will typically result in the replacement and dissemination of all $O\left(\log _{2} n\right)$ vertices on the path between the root certificate and the vertex containing the mutated mapping.

Since the transfer of a single vertex to a client can have a latency of tens to thousands of milliseconds, it is important to minimize the number of transfers. Related techniques have been recently explored by Goodrich, Tamassia, et al.[10, 8, 19]. These approaches utilize either skiplists or self-balancing search trees of logarithmic depth. However, skiplists require that clients transfer two-to-three times more vertices than Fern's trie. Self-balancing search tress have similar depth, but rebalancing operations require complicated algorithms and the structural changes required to maintain balance reduces the utility of caches, and thus can result in the replacement and transfer of more tree vertices.

\section{Approach}

A Fern dictionary's internal structure is a binary Merkle-trie with path compression. Like the similarly authenticated authorization framework suggested by Tamassia et. al. in [19], vertices from Fern's Merkle-trie are suitable for distribution by peer-to-peer (P2P) Content Distribution Networks (CDNs) to clients who construct and maintain VOs for mappings on which they depend.

As illustrated in Figure 1, Fern's trie is a binary prefix tree whose internal vertices with no branching are eliminated. Name:value mappings are stored in leaf vertices whose search key is equal to the name's SHA-1 hash. Following the model of a Merkle-tree[14], internal nodes also contain SHA-1 hashes of each immediate descendant (these hashes are not indicated in this figure). The incorporation of these hashes permits clients who possess valid copies of internal vertices to validate the integrity of their descendants'. The current root 
and its hash is stored in a root certificate signed with the originator's public key using GPG's DSA cipher.

By creating a Fern dictionary, an originator serves as a Certification Authority for clients who depend on mappings stored within it. A Fern verification object (VO) for a particular name corresponds to a search path containing its mapping. If the name is stored within the trie, it will terminate in a leaf containing its mapping. Otherwise, it will contain a search path that demonstrates that the referenced name is not in the trie.

Our initial implementation of Fern publishes through the CoralCDN self-organizing and locality-aware content distribution network. Coral serves as a scalable autonomic proxy-cache for frequently-referenced vertices. Clients obtain vertices required to construct or update VOs from coral and thus avoid transferring the entire trie structure. This helps to minimize network congestion that arises from distributing vertices. Furthermore, client caching is exploited, thus eliminating the need to obtain the same vertex multiple times.

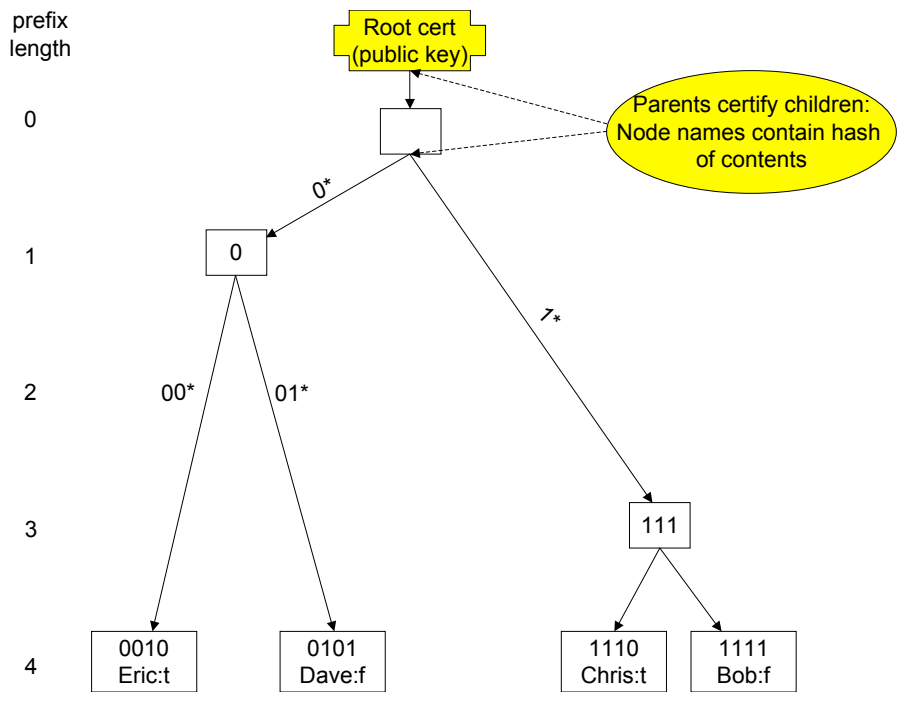

Figure 1: A Fern trie for 4-bit keys containing leaves (0010, 0101, 1110, and 1111). The leaves contain mappings of names to groups. Internal nodes are labeled by their corresponding key prefix and edges denote the bits that they match.

\subsection{Authentication with Fern}

Figure 1 depicts a simplified example of the structure of a Fern trie. In contrast to Fern, which uses 160-bit SHA-1 hash digests as search keys, the simplified trie depicted in Figure 1 only uses four-bit search keys. Name:value mappings are stored in leaf vertices with search-keys corresponding to the name's hash digest. In this figure, the name "Bob" (mapped to $f$ ) is depicted as having a hash equal to 1111.

Routing decisions within this trie are made one bit at a time, in decreasing order of significance. Each internal vertex is labeled with the search prefix corresponding to the common prefix shared by all its descendants. Vertices within tries that have no branching are collapsed as is illustrated by the absence of vertices corresponding to the prefixes $1,10,11$, and 110 .

A client with interest in an Id's mapping stored within Fern obtains the set of trie nodes that comprise the path from the root to the desired leaf. Monte Carlo experimenets indicate that leaves within Fern's trie are generally at a depth of $1.1 \log _{2} n$ where $\mathrm{n}$ is the number of mappings stored within the trie.

A leaf vertex with search key $k$ stores a name:value mapping where $k$ is the name's SHA-1 cryptographic hash. Each internal vertex of a Fern authorization trie contains node identifiers (NIDs) of its children. NIDs are used as a locator by clients requesting particular nodes. Like SFS-RO's[6] self-certifying pathnames, Fern's NIDs include cryptographic hashes of the referenced node's contents and are used to verify the integrity of a nodes obtained from insecure channels. 
As illustrated at the top of Figure 1, the Fern CA periodically publishes a signed, time-limited root certificate using asymmetric cryptography. Fern clients know the CA's asymmetric public-key identity and use it to determine the integrity of root certificates. Every internal node of a Fern trie contains NIDs of its immediate descendants, and NIDs contain cryptographic hashes of the nodes they reference. Thus the full path of trie nodes from a root certificate to a leaf node can be validated.

A VO for a referenced identifier, $I$, contains all Fern-nodes between the Fern-root and $I$. A full certification path can be included within the payload of a single message transmitted from one Fern client to another. Alternatively, since each internal node of a Fern-trie contains both search guides and the NIDs of immediate children, a Fern client can obtain a certification path for I by obtaining intermediate nodes as it traverses the path from the root toward the leaf node containing I.

A search-path that comprises a complete VO can be transmitted directly between clients, or instead be constructed by a client that recursively searches for the trie leaf containing the required name:value mapping.

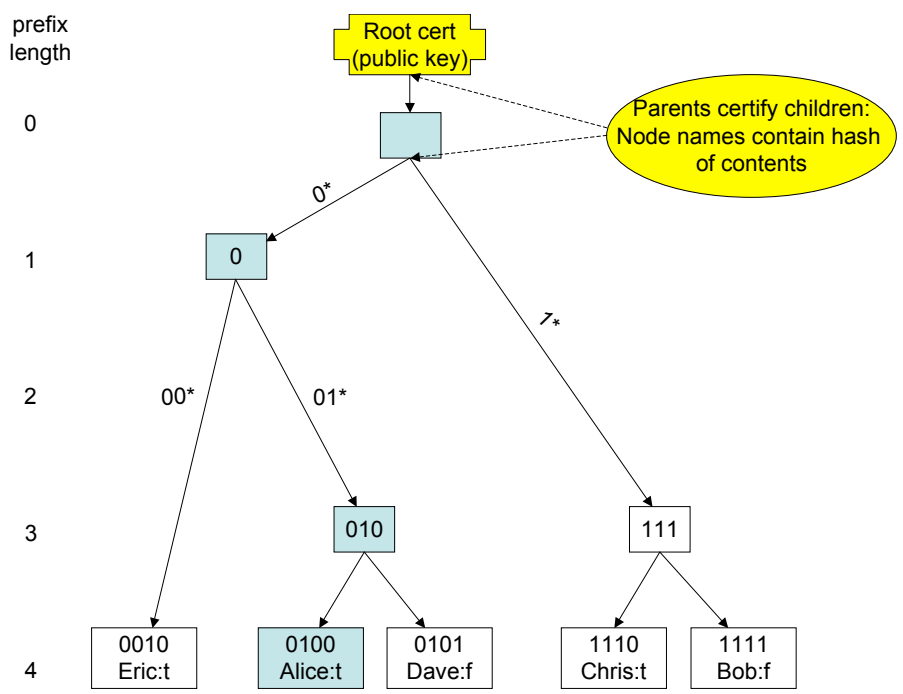

Figure 2: Inserting node 0100 requires inserting a new internal node 010 and updating all ancestor nodes (shaded) along the path.

\subsection{Trie Insertion}

Figure 2 illustrates modifications the the trie of Figure 1 after the insertion of a mapping for "Alice" to "t". Observe that this insertion also required the creation of a new internal vertex corresponding to prefix "010." Since each internal vertex contains the cryptographic hash of its children, all of Alice's ancestors will be updated as is indicated by shading in this figure. Since a new NID will be used to identify each of these updated vertices, they will be distributed by the $\mathrm{CDN}$ as replacement rather than as updated values.

Note that clients will be unaware of any updates until the next time a root certificate is issued that references the replacement root. Our prototype implementation of Fern generates a new root certificate every five minutes, and thus updates are grouped into five minute epochs.

\subsection{Interactions Between Clients}

Assume Bob and Alice are motivated to enter into a trust-sensitive relationship for which their Fern VOs can serve as supporting evidence. It is likely to be most efficient for them to exchange their complete VOs at the time the relationship is established.

Since this relationship may be prolonged, Alice will be motivated to monitor Bob's authorization and thus will need to update his VO every time the trie root is modified. Since a prolonged relationship may 
extend beyond the current root certificate's lifetime, additional vertices may be required to continue their mutual authorization. Our implementation of Fern automatically obtains vertices needed to update VOs representing such continuing relationships from the CDN and thus exploits the CDN's ability to scalability distribute online content in a locality-sensitive manner.

\section{Comparison with Other Cacheable Authenticated Dictionaries}

Several designs for authenticated dictionaries with equivalent asymptotic complexity have been proposed. In this section, we examine their expected cost and argue that the constants matter because latency of authenticated dictionary operations that rely on distributed caches to distribute vertices is heavily dependent on the latency of necessarily serialized communication.

As discussed in Section 3.3, we observe that the determination and maintenance of authorization are important operations for clients of authenticated dictionaries. In both cases, a client's challenge is to repeatedly obtain and validate the subset of a dictionary's current vertices needed to maintain VOs that authorize relationships on which the client relies.

A VO for a particular mapping stored within an authenticated dictionary can be quickly generated if all needed vertices are available from a nearby dictionary. However, if the dictionary is large, connectivity is limited, or worse connectivity varies dynamically, it may be impractical to distribute the entire dictionary to proxies with good connectivity to clients.

Fern uses an alternative approach of distributing a dictionary's data structures incrementally through the use of an autonomic content distribution infrastructure (such as a DHT). In such a deployments, each successive vertex in a VO's transitive chain must be obtained sequentially.

As observed by Tammasia and Triandopolos, the expected time to acquire a dictionary node stored within a DHT composed of $P$ hosts will require $O(\log P)$ pairwise communications that must be serialized. Since each pairwise communication can take dozens to hundreds of milliseconds, communication latency is likely to dominate the time required to assemble a VO.

Since the computational cost related to construct and verify a VO is small, the time required by a client to assumable or update a $\mathrm{VO}$ will be dependent on the the number of nodes that this client must obtain from the content distribution infrastructure.

Tamassia, Goodrich, Winsboro, and others identified skiplists[17] and various self-balancing trees[16, 3] as having the following desirable properties for the underlying data structures used for cacheable and updatable authenticated dictionaries.

- Logarithmic expected leaf node depth with narrow variance. This ensures that the number of vertices within a VO is logarithmic in the number of words stored within the dictionary

- Localization of tree update operations. Rotation and other tree-rebalancing operations disrupt the tree structure and thus require replacement (and transmission) of tree vertices that might not otherwise be affected by a particular tree update operation.

A Fern VO for a word stored at depth $d$ contains $d$ dictionary vertices. Monte Carlo experiments indicate that, within a very narrow variance, the expected value for $d$ is $1.1 \log _{2} n$ for $n>2^{13}$. Furthermore, as described in Section ??, update operations associated with a word stored within a leaf of a Fern trie are localized to that leaf and its (approximately $1.1 \log _{2} n$ ) ancestors.

In [10], Goodrich, Tammasia, et al. identify skiplists as suitable structures for constructing authenticated dictionaries in a distributed environment. While skiplists share Fern's desirable property of not requiring rebalancing, we observe that a client must collect a greater number of vertices when assembling a skiplistbased VO than a Fern VO: The expected number of skiplist vertices that must be examined when assembling a VO from a dictionary containing $n$ words is $3 \log _{2} n$, and the expected number of vertices within a skiplist that will be modified when an entry is updated is $2 \log _{2} n$. As described in Section 4.1, Fern's expected value of $1.1 \log _{2} n$ for the number of nodes to update compares very favorably with skiplists.

In [19], Tamassia and Triandopolos identify red-black and $\mathrm{BB}[\alpha]$ as suitable dictionary structures for constructing authenticated dictionaries whose vertices are, like Fern, distributed by an autonomic DHTbased cache. These self-balancing trees utilize rotations to guarantee that the search-structure remains approximately balanced. While these rotations are infrequent, they will increase the number of vertices 
that are replaced and thus increase network communication required to update VOs not associated with the updated mapping.

The advantage that Fern's Merkle-trie achieves over self-balancing trees and skip lists arises from the ability of a good hash function (e.g. SHA-1) to evenly distribute a set of elements over an ordered range which is large compared to the number of elements being distributed. Each data element is hashed to the range $0 \ldots 2^{160}$, where each value corresponds to a possible leaf of Fern's Merkle-trie. The specific values to which data elements are hashed become the actual leaves of Fern's Merkle-trie. It is because of the nature of the hashing function that the Merkle-trie is created in a well-balanced manner, without any dynamic interventions. This, massive virtual hash table structure is usable because it allocates memory for nodes in a lazy fashion. No node is allocated unless it is an actual leaf or if both of its children are either actual leaves or ancestors of actual leaves. Thus, as is common in tries, some edges will be compressed and further reduces the memory required for this trie, and reduces the number of vertices within a VO.

\subsection{Expected number of refresh queries}

The hash value of a Merkle-tries's leaf and all of its ancestors changes whenever the leaf's contents are updated. In this section, we consider the number of vertices that must be obtained by a client who is monitoring mappings stored within a set of $w=|W|$ watched leaves within a dictionary storing a total of $n$ mappings in the event that $u=|U|$ leaves are updated.

Let $c=|U \cap W|$ be the number of vertices in $W$ whose mappings have changed.

Below, we informally demonstrate how the cases where $w \leq u$ and $u \leq w$ are reducible to each other. Throughout both analyses, we will be making the approximation of a well balanced trie at all levels. Our empirical studies[?] indicate taht this is a good approximation, especially as $w, u$ and $n$ become large. We begin with an informal overview and then provide arguments that constrain the constants.

\subsection{Case 1: $w \leq u$}

As illustrated in Figure 3, our analysis divides the trie into three regions. The upper region, which extends to depth $\left\lfloor\log _{2} w\right\rfloor$ has approximately $w$ vertices at its (approximate) lower edge. We assume that our hash function has uniformly distributed keys, and thus assume that most of these (approximately) $w$ vertices are ancestors to the $w$ leaves mapping members of $W$. Since $u \geq w$, it is likely that most of these (approximately) $w$ vertices members are also ancestors of the members of $|U|$. Thus our client is likely to require updates for all (approximately) $2 w-1$ vertices in the upper region.

The middle region of this trie extends to depth $\left\lfloor\log _{2} u\right\rfloor$. Like our analysis of the upper region, it is likely that most vertices above depth $\log _{2} u$ contain nodes that are ancestors of the $u$ updated leaves and thus most vertices in this region are likely to have been updated. However, only $w$ paths of length $\left\lfloor\log _{2}(u / w)\right\rfloor$ through this middle region are likely to be ancestors of vertices being watched,and thus only $w\left\lfloor\log _{2}(u / w)\right\rfloor$ of these vertices are likely to be needed by the client.

The lower region extends down from (approximately) level $\left\lfloor\log _{2} u\right\rfloor$.and thus has approximately $\lceil\log (n / u)\rceil$ levels. Since we assume that clients previously cached unchanged vertices leading to members of $W$, we expect that clients' searches will terminate near to the top of this region for most of the $w-c$ paths that do not lead to modified leaves. However, the approximately $c\left\lceil\log _{2}(n / u)\right\rceil$ vertices along the $c$ paths to leaves that are both watched and updated will be obtained by clients.

Thus we expect that approximately $(2 w-1)+w\left\lfloor\log _{2}(u / w)\right\rfloor+c\left\lceil\log _{2}(n / u)\right\rceil$ nodes that will require updating. In practice, the shift from one section to the next may not occur exactly at the same level on every path, but these small variations do not significantly not change our estimates.

\subsection{Case 2: $u \leq w$}

As with Case 1, we anticipate that most of the $2 u-1$ vertices in the upper region will be ancestors to both watched and updated leaves, and thus will be obtained by clients.

Similarly, we anticipate that while most vertices in the middle region (with a lower edge containing $w$ vertices) will be watched, only $u$ paths will continue through to the bottom of this section of height 


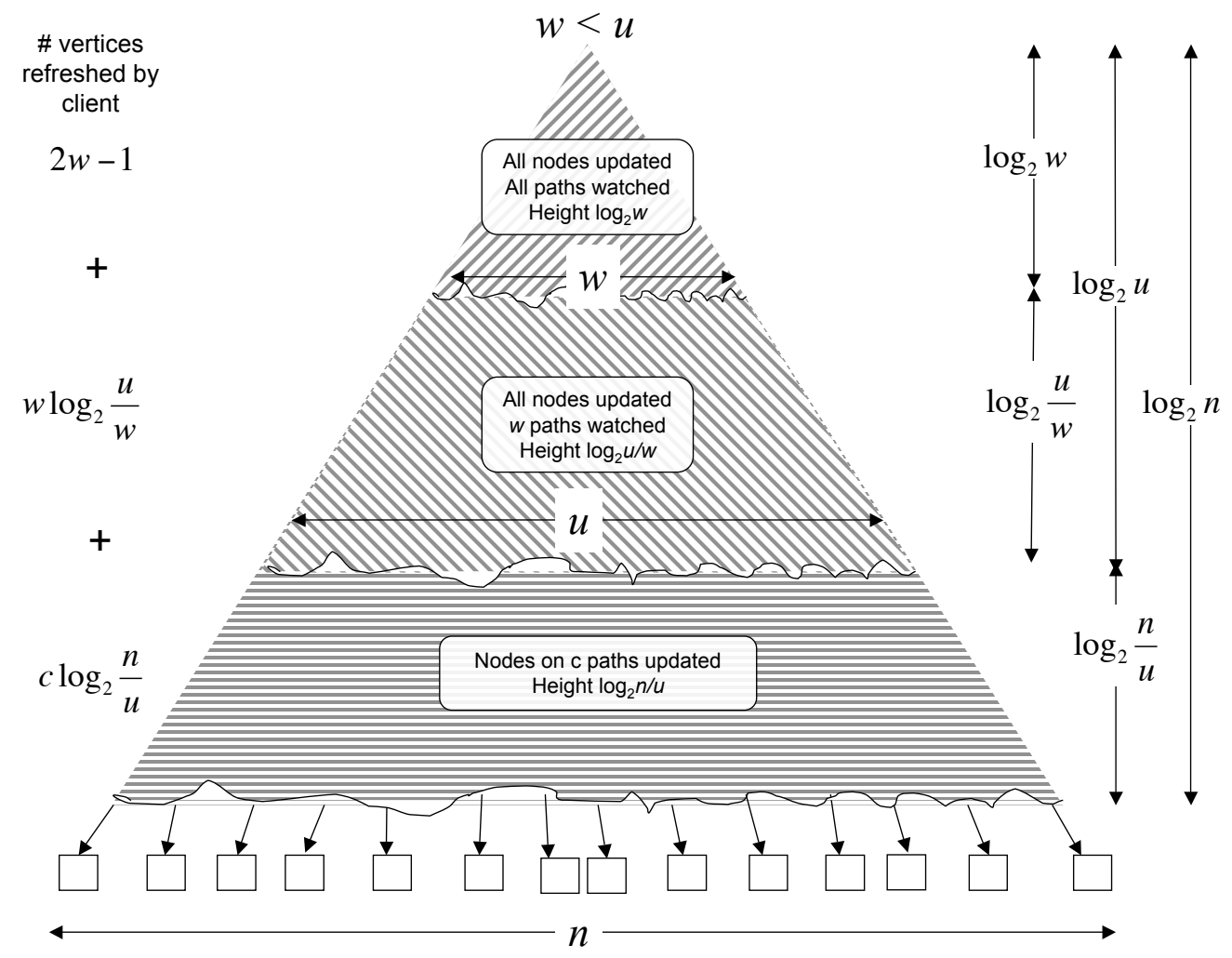

Figure 3: Illustration depicting our analysis of the number of nodes that need to be updated when the number of watched data entries is smaller than the number of updated data entries 


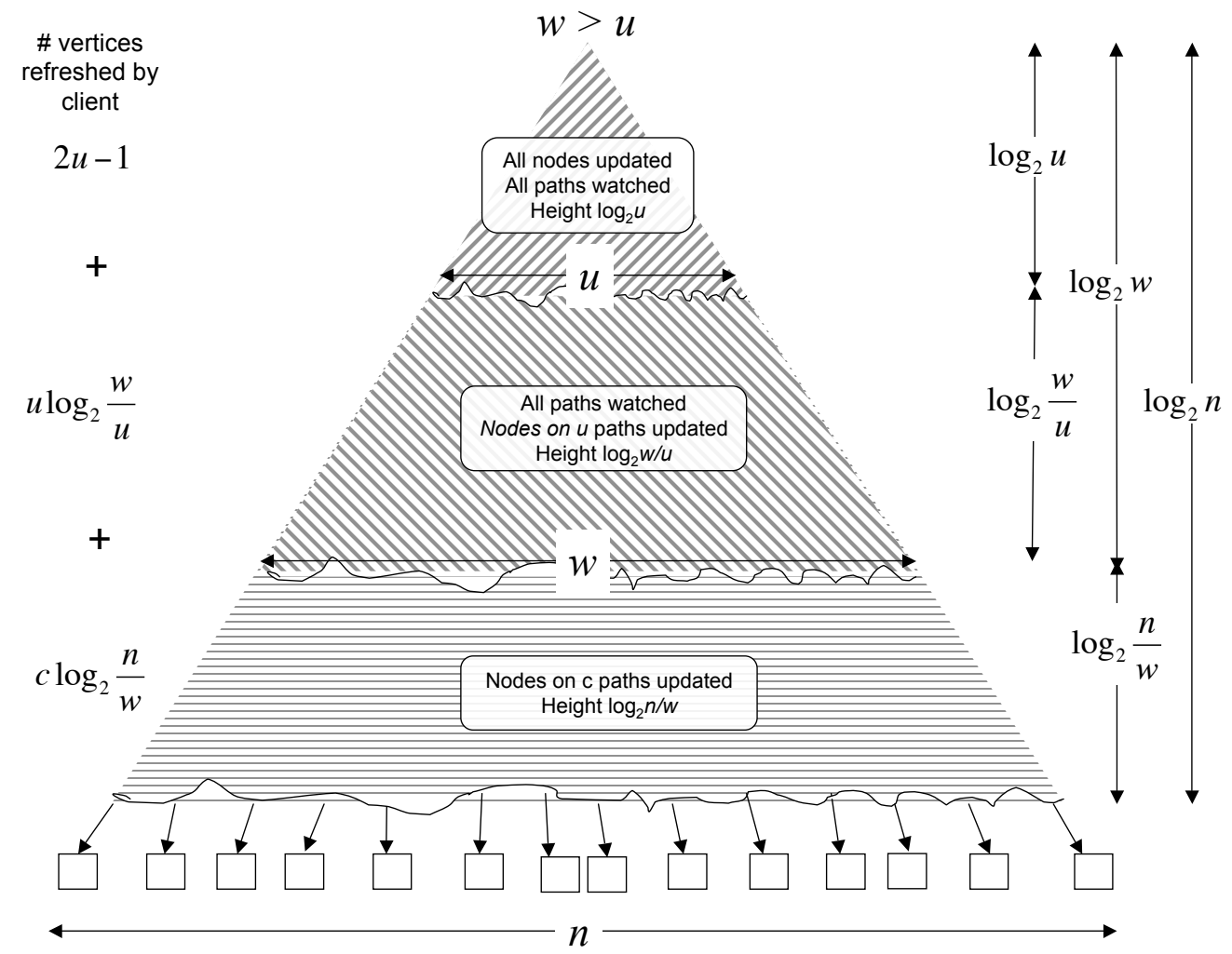

Figure 4: Illustration depicting our analysis of the number of nodes that need to be updated when the number of watched data entries is greater than the number of updated data entries 
$\left\lfloor\log _{2} w / u\right\rfloor$ towards updated leaves, and that the other $w-u$ paths will terminate near its upper boundary. Thus, approximately $\left\lfloor u \log _{2} w / u\right\rfloor$ of its vertices will be updated and therefore obtained by clients.

Thus $w$ paths through the lower region will be watched. However, only $c$ of them will lead to leaves in $U$ and thus we expect that approximately $c\left\lfloor\log _{2} n / w\right\rfloor$ vertices in this region will be obtained by clients.

Finally using the same arguments, approximately $(2 u-1)+w\left\lfloor\log _{2}(w / u)\right\rfloor+c\left\lceil\log _{2}(n / w)\right\rceil$ vertices will be obtained by clients. They approach approach the same value for the case $w=u$, which also corresponds to the situation where the middle term of both expressions becomes 0 .

As stated above, the expected number of nodes a skiplist based dictionary would need to update is $2 \log _{2} n$, for the case $c=u=w=1$. For this case, our expression reduces to $1+\log _{2}(n)$. Once again, we see Fern achieves a significant advantage when one observes the constants within the asymptotic expressions.

\subsection{Tightening The Bounds}

When a leaf in a Merkle trie has its contents updated, its hash value is changed. This change propagates to all the ancestors of this leaf causing their hash values to change. Suppose a dictionary, that is stored in the leaves of a Merkle trie, contains $n$ entries, of which $u$ are updated. Suppose a user is watching $c$ entries that are changed and $w$ entries that are not changed. Let $r$ be the number of refreshes that are caused by these updates. Assume that all entries of the trie are generated randomly and independently and that all updates and watched entries are generated randomly and independently among the entries of the trie. How does the number of expected nodes that need to be refreshed $E(r)$ increase as the number of updated nodes grows?

We analyze this problem assuming no path compression in the trie other than compression at the leaves. We believe compression would not change the analysis assuming $n$ is much larger than $u$.

Let's first look at the case $c=0$ and $w=1$. We show that the expected number of nodes, that need to be refreshed grows as $\Theta(\lg (u))$ within a tight set of bounds.

The expected number of refreshes $E(r)$ corresponds to the longest common prefix between the hash of the watched entry and the hash of an updated entry. Let $X_{i}$ be a random variable that is 0 if the longest common prefix is $<i$ and 1 if it is $\geq i$. Then, $X=\sum_{i=1}^{\infty} X_{i}$ is a random variable equal to the longest common prefix. We have that $E(X)=E\left(\sum_{i=1}^{\infty} X_{i}\right)=\sum_{i=1}^{\infty} E\left(X_{i}\right)=\sum_{i=1}^{\infty} P\left(X_{i}\right)$. We also have $P\left(X_{i}\right)=1-\left(1-1 / 2^{i}\right)^{u}$.

While an exact solution to the summation seems daunting, we offer a reasonably tight approximation.

Lower bound. $\sum_{i=1}^{\infty} 1-\left(1-1 / 2^{i}\right)^{u} \geq \sum_{i=1}^{\lg u} 1-\left(1-1 / 2^{i}\right)^{u} \geq \sum_{i=1}^{\lg u} 1-\left(1-1 / 2^{\lg u}\right)^{u}=\sum_{i=1}^{\lg u} 1-(1-$ $1 / u)^{u} / \operatorname{leq} \sum_{i=1}^{\lg u} 1-1 / e=(1-1 / e) \lg u$.

Upper bound. $\sum_{i=1}^{\infty} 1-\left(1-1 / 2^{i}\right)^{u}=\sum_{i=0}^{\infty} \sum_{j=1}^{\lg u} 1-\left(1-1 / 2^{i \lg u+j}\right)^{u}=\sum_{i=0}^{\infty} \sum_{j=1}^{\lg u} 1-\left(1-1 /\left(u^{i} 2^{j}\right)\right)^{u} \leq$ $\sum_{i=0}^{\infty} \sum_{j=1}^{\lg u} 1-\left(1-1 /\left(u^{i+1}\right)\right)^{u}=\lg u \sum_{i=0}^{\infty} 1-\left(1-1 /\left(u^{i+1}\right)\right)^{u} \leq \lg u \sum_{i=0}^{\infty} 1-\left(1-u /\left(u^{i+1}\right)\right)=\lg u \sum_{i=0}^{\infty} 1 / u^{i}=$ $\left(1+\frac{1}{u-1}\right) \lg u$.

So we now have $(1-1 / e) \lg u \leq E(r) \leq\left(1-\frac{1}{u-1}\right) \lg u$. Although we don't have a formal proof, some further investigation leads us to believe that $E(r) \in \lg u \pm o(\lg u)$.

Now, consider the case $c=0, w \geq 1$ and $u>w$. We provide an informal argument that the expected number of refreshes should be $\Theta(w \lg (u / w))$. The argument is still informal in that it requires $u>>w$ in a way that depends on the hidden constant in the asymptotic notation and that we didn't spell out explicitly.

What we try to prove corresponds to the previous result when $w=1$. The argument works by induction on $w$. Assume we have $w-1$ watched. The expected number of refreshes is $r_{\text {old }}+r_{\text {new }}-r_{\text {common, where }}$ $r_{\text {old }}=$ the number of refreshes generated by the $w-1$ values, $r_{\text {new }}=$ number of refreshes generated by the new value and $r_{\text {common }}=$ the number of refreshes that are common.

Let $R(w, u)$ be the number of refreshes required when $u$ updates are made and $w$ words are watched We want to show $E(R(w, u)) \leq a w \lg (u / w)$ for some constant $a . E(R(w, u)) \leq E(R(w-1, u))+\lg u$. By induction hypothesis, this is

$a(w-1) \lg (u /(w-1))+\lg u$

$=a w \lg (u / w)+a w \lg (w /(w-1))-a \lg (u /(w-1))+\lg (u)$

$=a w \lg (u / w)+\lg \left(w^{a w} /\left((w-1)^{a(w-1)} u^{a-1}\right)\right)$.

So, when $a$ is a reasonably large constant and $u$ is reasonably larger than $w$,

$u \geq(w /(w-1))^{a(w-1) /(a-1)} w^{a /(a-1)}$ and we have $E(R(w, u)) \leq a w \lg (u / w)$.

When $c>0$, we need to add the whole refresh path for the changed values minus those from that path that were already refreshed. It comes out to $w \lg (u / w)+c(\lg n-\lg w)$. 


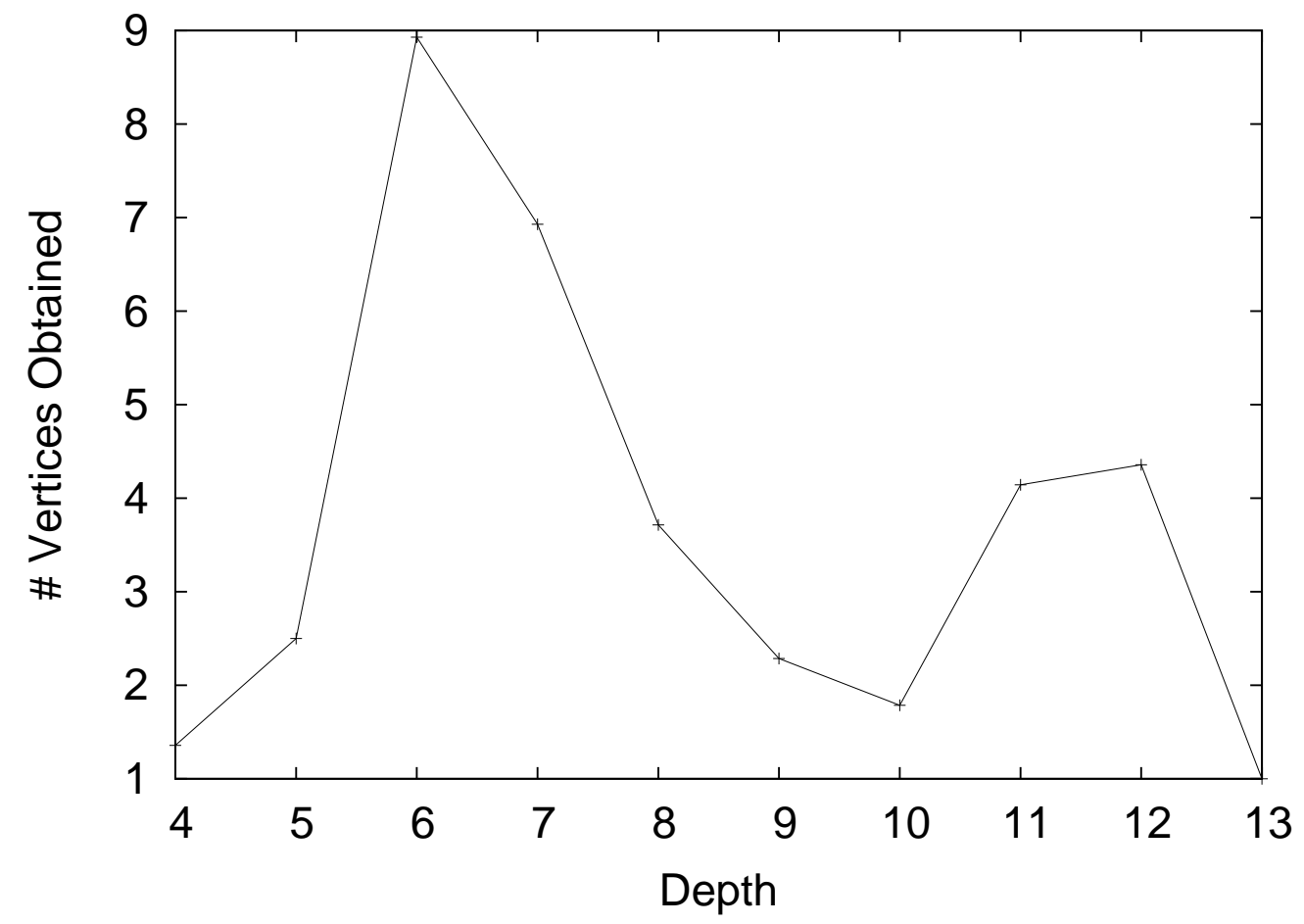

Figure 5: Average Depth of Refresh Queries. As predicted by the analytical model, refresh queries tend to terminate around depths $\log _{2} u=7$ and $\log n=11$. 


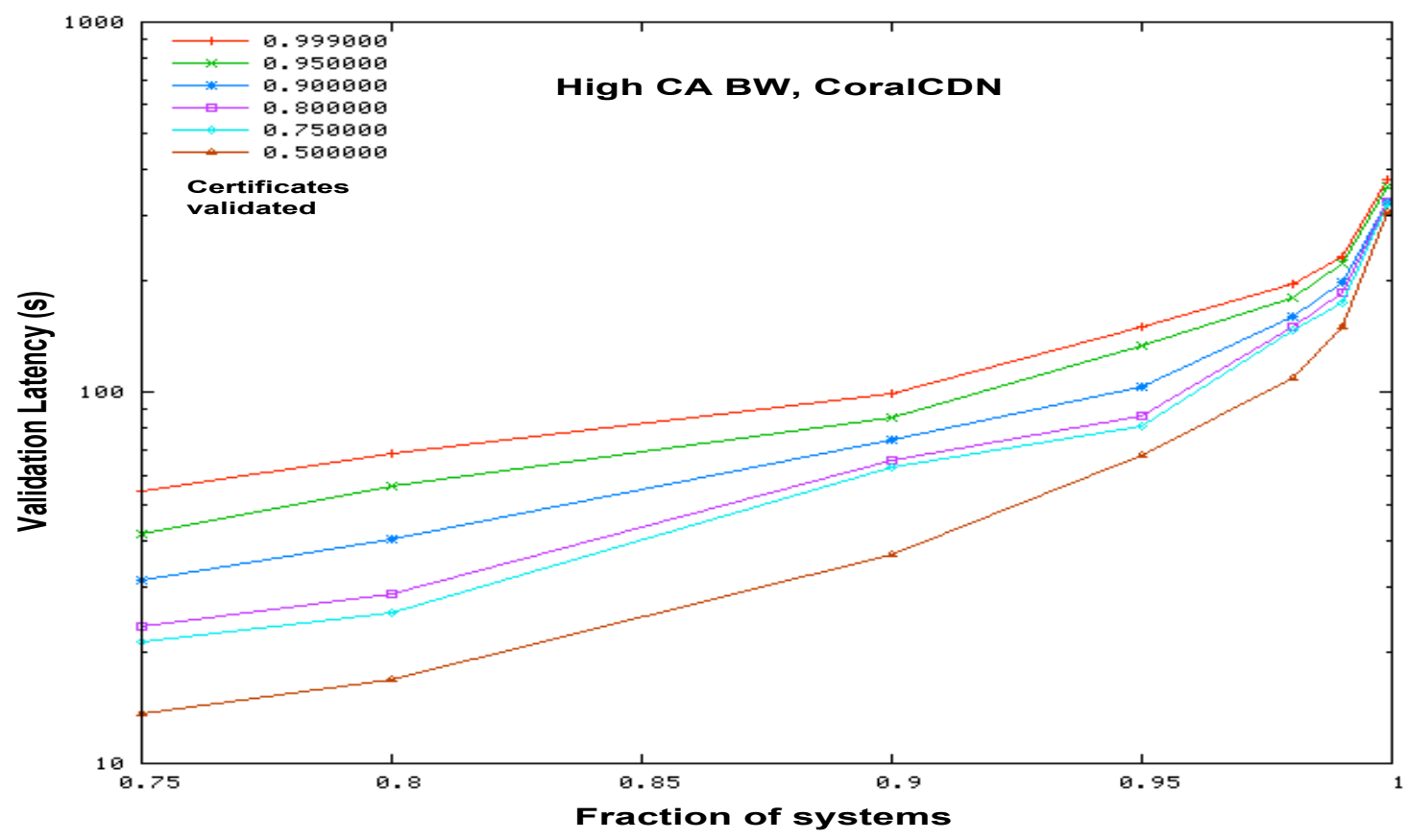

Figure 6: In-vivo experiment upon Planetlab.

Figure 5 provides empirical evidence supporting our analytical model of refresh queries. In this experiment, $k=2058, w=37, u=130$. Reference patterns correspond to pairs of systems authenticating each other in order to maintain routing tables for a Kademlia[13] DHT. Plots indicate the average number of Fern vertices obtained for clients who obtained $c=8$ updated nodes during this experiment. In these experiments, the average number of vertices obtained by clients is 154 . Our analytical approximation prediction (Case 1) is 146. Surprisingly, this observed average number of refreshed vertices is within $2 \%$ of the modeled value. ${ }^{4}$

We have begun evaluation of Fern upon the Planetlab[1] global distributed testbed. Figure 6 was generated in an experiment with approximately 700 hosts, each monitoring approximately sixty mappings chosen with distributions that mimic routing tables in a Kademlia[13] distributed hash table. Each time the dictionary was updated, two hundred randomly selected mappings were changed. Each plot depicts the fraction of mappings obtained by each host. The vertical axis represents latency in seconds, the horizontal axis represents the fraction of systems.

\section{Synopsis}

Fern is an updatable cryptographically authenticated dictionary. Unlike conventional approaches that require provisioning of proxies that maintain full duplicates of the dictionary structure at untrusted proxies, Fern utilizes an scalable and locality-aware autonomic content distribution network that incrementally distributes portions of the dictionary as required by clients.

Fern's use of an autonomic CDN makes it suitable for dynamic and Peer-to-Peer environments where dedicated resources may not be available. Furthermore, Fern can also be used to fortify the same CDN on which it relies against disruption from unauthorized systems.

A range of tree structures have been proposed for implementing updatable dictionary structures. While most of these algorithms have similar asymptotic behavior, the number of vertices that must be transferred to a client incrementally looking up a mapping stored within authenticated dictionary structures varies dramatically. Since the communication latency related to transferring nodes to clients will dominate the

\footnotetext{
${ }^{4}$ We have not yet performed a sensitivity study on this result, but this will be complete prior to the author notification date and included in the final version of this paper.
} 
latency of client operations, these constants do matter. Our experiments indicate that Fern's randomized trie requires that substantially fewer vertices be transferred to clients than skiplists, and a comparable number with more complicated self-balancing trees.

Furthermore clients may need to monitor the stability of mappings (not) stored within an authenticated dictionary. Since Fern achieves an approximately balanced structure without rebalancing, clients can efficiently utilize caching of previously obtained vertices, thus minimizing the number of vertices that must be transferred when a dictionary update is distributed.

Initial experiments conducted upon the Planetlab[1] global distributed testbed indicate that the CoralCDN provides similar performance to a large number of clients who have a limited bandwidth to a Fern

originator. In addition, our analysis of the number of vertices that must be transferred to a client monitoring a set of mappings stored within a Fern trie approximates observed operation.

\section{References}

[1] Planetlab: An open platform for developing, deploying, and accessing planetary-scale services. http://planet-lab.org.

[2] Anagnostopoulos, A., Goodrich, M. T., and Tamassia, R. Persistent authenticated dictionaries and their applications. In Lecture Notes in Computer Science, Proc. Information Security Conference (ISC) (2001), vol. 2200, Springer-Verlag, pp. 373-393.

[3] BAYER, R. Symmetric binary b-trees: Data structure and maintenance algorithms. Acta Inf. 1 (1972), 290-306.

[4] Ellison, C. M., Frantz, B., Lampson, B., Rivest, R., Thomas, B. M., and Ylonen, T. SPKi Certificate Theory. IETF RFC 2693, 1998.

[5] Freedman, M., Freudenthal, E., and Mazieres, D. Democratizing content publication with coral. In Proc. NSDI 2004 (2004).

[6] Fu, K., KaAshoek, M. F., and Mazières, D. Fast and secure distributed read-only file system. Computer Systems 20, 1 (2002), 1-24.

[7] Garfinkel, S., and Spafford, G. Practical UNIX and Internet Security. O'Reilly and Associates, Inc., 1996.

[8] Goodrich, M., And Tamassia, R. Efficient authenticated dictionaries with skip lists and commutative hashing, 2000.

[9] Goodrich, M., Tammassia, R., and Schwerin, A. Implementation of an authenticated dictionary with skip lists and commutative hashing. In DARPA Information Survivability Conference and Exposition II (DISCEX II) (2001).

[10] Goodrich, M. T., Shin, M., Tamassia, R., and Winsborough, W. H. Authenticated dictionaries for fresh attribute credentials. In iTrust (2003), P. Nixon and S. Terzis, Eds., vol. 2692 of Lecture Notes in Computer Science, Springer, pp. 332-347.

[11] Li, N., Mitchell, J. C., And Winsborough, W. H. Design of a role-based trust management framework. In Proc. 2002 IEEE Symposium on Security and Privacy (May 2002), IEEE Computer Society Press, pp. 114-130.

[12] Martel, C., Nuckolls, G., Devanbu, P., Gertz, M., Kwong, A., And Stubblebine, S. A general model for authenticated data structures, 2001.

[13] Maymounkov, P., And Mazieres, D. Kademlia: A peer-to-peer information system based on the xor metric. In Proc. IPTPS02 (2002). 
[14] Merkle, R. C. A certified digital signature. In Advances in Cryptology - CRYPTO '89, G. Brassard, Ed., vol. 435. Springer-Verlag, 1990, pp. 218-238.

[15] Myers, M., Ankney, R., Malpani, A., Galperin, S., and Adams, C. X.509 Internet Public Key Infrastructure Online Certicate Status Protocol. IETF RFC 2560, 1996.

[16] Nievergelt, J., And Reingold, E. M. Binary search trees of bounded balance. SIAM J. Comp. 2 (1973), 33-43.

[17] Pugh, W. Skip lists: A probabilistic alternative to balanced trees. In Workshop on Algorithms and Data Structures (1989), pp. 437-449.

[18] Rivest, R. L., and Lampson, B. SDSI - A simple distributed security infrastructure. In Proc. of CRYPTO'96 (1996).

[19] Tamassia, R., and Triandopoulos, N. Efficient content authentication over distributed hash tables. Tech. rep., Brown University, 2005. 